\title{
TRADUCCIÓN ACCESIBLE: AVANCES DE LA NORMA ESPAÑOLA DE SUBTITULADO PARA SORDOS UNE 153010:2012
}

\author{
por CARMEN CUÉLLAR LÁZARO
}

(Universidad de Valladolid)

\begin{abstract}
Resumen
El año 2003 supuso en España un punto de inflexión en la estandarización de los subtítulos para sordos al publicarse la norma UNE 153010:2003 Subtitulado para personas sordas y personas con discapacidad auditiva. Subtitulado a través del teletexto, elaborada por la Asociación Española de Normalización y Certificación (AENOR). Casi una década después, en 2012, esta norma es anulada y sustituida por la norma UNE 153010:2012. Subtitulado para personas sordas y personas con discapacidad auditiva, que, con un carácter más general, contempla todos los escenarios de la comunicación audiovisual y tiene como objetivo establecer unos requisitos mínimos de calidad y homogeneidad en el subtitulado para personas sordas y personas con discapacidad auditiva. A través de un estudio contrastivo entre ambas normas observaremos los avances que se han llevado a cabo y destacaremos aquellos aspectos más relevantes que debe tener en cuenta el subtitulador como creador de contenidos audiovisuales accesibles, consciente de que la finalidad de su trabajo es la eliminación de barreras, en este camino hacia la igualdad de oportunidades para este colectivo.
\end{abstract}

Palabras clave: traducción audiovisual; subtitulado para sordos; accesibilidad; norma UNE 153010

\begin{abstract}
In Spain, 2003 marked a turning point in the standardization of subtitling for the deaf with the publication of the UNE norm 153010:2003 "Subtitling for the Deaf and the Hard of Hearing. Subtitling by tele text", developed by the Spanish Association of Normalization and Certification (AENOR). Nearly a decade later, in 2012, this norm was replaced with the UNE norm 153010:2012 "Subtitling for the Deaf and the Hard of Hearing" which, with a more general character, contemplates the full range of possible scenarios for audio-visual communication and aims to establish basic requirements in quality and homogeneity in the subtitling for the deaf and the hard of hearing. Through a comparative study of both norms, we will outline the advances that have been carried out within the field of subtitling. We will emphasize the essential aspects that a subtitler must bear in mind - as a creator of audio-visual accessible content - by being aware that the ultimate purpose of their work is the elimination of barriers in the way towards the realization of equality of opportunities for the members of the deaf community.

Keywords: audiovisual translation; subtitling for the deaf and hard of hearing; accessibility; UNE norm 153010
\end{abstract}




\section{Introducción}

En una sociedad cada vez más sensibilizada hacia los temas de accesibilidad, la eliminación de barreras supone un reto para conseguir la igualdad de todos los ciudadanos. En este sentido, el acceso a la información, la cultura y el entretenimiento es un requisito indispensable para lograr esa igualdad en el colectivo de personas sordas y con discapacidad auditiva.

La accesibilidad en los medios, desde una visión multidisciplinar, ha entrado ya plenamente en los Estudios de Traducción con la incorporación de investigación y formación en traducción accesible. ${ }^{1}$

A nivel normativo, España es uno de los países que lidera este proceso de sensibilización hacia la necesidad de una subtitulación dirigida al colectivo de personas sordas. ${ }^{2}$ Desde finales del siglo XX y comienzos del siglo XXI se han aprobado leyes y normas que contribuyen a eliminar los obstáculos con los que se encuentra la comunidad sorda en su acceso a los productos audiovisuales. Entre esos logros institucionales se encuentra la norma de estandarización del subtitulado para sordos (SPS) publicada en 2012 UNE 153010:2012 Subtitulado para personas sordas y personas con discapacidad auditiva elaborada por la Asociación Española de Normalización y Certificación (AENOR), que anula y sustituye la norma anterior UNE 153010: 2003 Subtitulado para personas sordas y personas con discapacidad auditiva. Subtitulado a través del teletexto.

Este estudio se marca como objetivo realizar un análisis comparado de ambas normas para observar los avances que se han llevado a cabo en estos años, destacando aquellos aspectos más relevantes que debe tener en cuenta el subtitulador como creador de contenidos audiovisuales accesibles. Los avances en la normativa deben favorecer la tarea del subtitulador, consciente de que la finalidad de su trabajo es la eliminación de barreras en el camino hacia la igualdad de oportunidades para este colectivo.

\section{Contexto en el que se desarrolla la norma $U N E$ 153010:2012}

En 2003 se celebró el Año Europeo de las personas con discapacidad, lo que impulsó que en España se aprobara la Ley 51/2003, de 2 de diciembre, de igualdad de oportunidades, no discriminación y accesibilidad universal de las personas con discapacidad. ${ }^{3}$ Este mismo año supuso un punto de inflexión en la estandarización del subtitulado para sordos al publicarse en España la norma UNE 153010:2003, de la que hemos hablado en el apartado anterior, que se centraba en el subtitulado para

1 Sobre el estado de la formación en accesibilidad audiovisual cfr. Toni BADIA - Anna MATAMALA, "La docencia en accesibilidad en los medios", Trans. Revista de traductología II, 2007, pp. 61-71 y Carmen CUÉLLAR LÁZARO, "El subtitulado para sordos en España y Alemania: estudio comparado de los marcos normativos y la formación universitaria", Revista Española de Discapacidad 4/2, 2016, pp. 143-162.

2 Crf. Josélia NEVES - Lourdes LORENZO GARCÍA, "La subtitulación para s/Sordos, panorama global y prenormativo en el marco ibérico”, Trans. Revista de traductología II, 2007, p. 100.

3 El Consejo de Ministros aprueba en 2003 el I Plan Nacional de accesibilidad 2004-2012. Por un nuevo paradigma, el Diseño para Todos, hacia la plena igualdad de oportunidades, [consultado 20 de octubre de 2016]. Accesible de: http://www.sidar.org/recur/direc/legis/ipna2004_2012.pdf 
personas sordas y con discapacidad auditiva a través del teletexto únicamente. Casi una década después, como ya hemos adelantado, esta norma es anulada y sustituida por la norma UNE 153010:2012 Subtitulado para personas sordas y personas con discapacidad auditiva, que con un carácter más general, contempla todos los escenarios de la comunicación audiovisual, marcándose como objetivo establecer unos requisitos mínimos de calidad y homogeneidad en el subtitulado para este colectivo. ${ }^{4}$

Entre ambas, en 2007, se publica la LEY 27/2007, de 23 de octubre, por la que se reconocen las lenguas de signos españolas y se regulan los medios de apoyo a la comunicación oral de las personas sordas, con discapacidad auditiva y sordociegas. ${ }^{5}$ En su Artículo 24 se sientan las bases para la creación del Centro Español del Subtitulado y la Audiodescripción (el CESyA).

La Ley 7/2010, de 31 de marzo, General de la Comunicación Audiovisual (LGCA) establece de manera detallada las obligaciones de emisión que han de cumplir los radiodifusores en relación a los tres servicios de apoyo a la comunicación necesarios para las personas con discapacidad sensorial: subtitulado, audiodescripción y lengua de signos. Todas las cadenas públicas y privadas de cobertura estatal y autonómicas están sujetas a las obligaciones que recoge esta ley. Su artículo 8 hace referencia a que las personas con discapacidad visual o auditiva tienen el derecho a una accesibilidad universal a la comunicación audiovisual, de acuerdo con las posibilidades tecnológicas. ${ }^{6}$

En este estudio nos centramos en la modalidad de subtitulado para personas sordas y con discapacidad auditiva. ${ }^{7}$ A través de un análisis comparado entre las normas de 2003 y 2012, observaremos los avances que se han llevado a cabo en estos años. ${ }^{8}$

4 A lo largo de este estudio nos referiremos a la norma publicada en 2003 también como AENOR 2003 y la de 2012 como AENOR 2012.

5 Publicada en el Boletín Oficial del Estado (BOE) núm. 255. Miércoles 24 octubre 2000, pp. 43251-43259.

6 Cfr. en CUÉLLAR LÁZARO "El subtitulado" la evolución del porcentaje real de emisión subtitulada y el porcentaje impuesto por la LGCA en los últimos años.

7 Además de las normas que hemos comentado, se han publicado en España en los últimos años, entre otras, las siguientes relacionadas con la discapacidad y la accesibilidad: UNE 153020:2005 Audiodescripción para personas con discapacidad visual. Requisitos para la audiodescripción y elaboración de audioguías; UNE 139802:2003 Accesibilidad del Software, anulada por la norma UNE 139802:2009 Accesibilidad del Software; UNE 139803:2004 Aplicaciones informáticas para personas con discapacidad. Requisitos de accesibilidad para contenidos en la Web, anulada por la UNE 139803:2012 Requisitos de accesibilidad para contenidos en la Web. UNE 139804:2007 Lengua de signos (LSE) en Redes Informáticas.

8 Una reseña histórica de la accesibilidad en los medios más extendidos y desarrollados: el subtitulado para personas sordas y la audiodescripción, en Pilar ORERO, "Visión histórica de la accesibilidad en los medios en España", Trans. Revista de traductología II, 2007, pp. 31-43. Cfr. asimismo el estudio de Ana Ma PEREIRA RODRÍGUEZ, "El subtitulado para sordos: estado de la cuestión en España", Quaderns 11, 2005, pp. 161-172 y NEVES - LORENZO, "La subtitulación", con un panorama global de la subtitulación para sordos en España y Portugal. Cfr. igualmente otros estudios sobre el SPS en España en Francisco UTRAY et al., "The Present and Future of Audio Description and Subtitlingfor the Deaf and Hard of Hearing in Spain”, Meta 54/2, 2009, pp. 248-263 y Lourdes LORENZO 


\section{Subtitulado convencional vs SPS}

A continuación comentamos algunos aspectos de interés de esta modalidad de traducción audiovisual, el subtitulado convencional, así como la variante destinada a personas sordas y con discapacidad auditiva.

En el proyecto europeo Comparative Subtitling Project del año 2000 se llevó a cabo un estudio comparado del subtitulado en 18 lenguas de 25 países diferentes. En él participaron un total de 48 instituciones diferentes de Europa y el resto del mundo y se puso de manifiesto las variaciones de parámetros y criterios en la práctica profesional del subtitulado. ${ }^{9}$

Desde los Estudios de Traducción, los investigadores coinciden con esta visión al afirmar que "No hace falta ver muchos programas subtitulados en cine o televisión, en España o en otros países, para darse cuenta de la falta de consenso armónico a la hora de implementar las convenciones formales que regulan la entrega de los subtítulos en pantalla". ${ }^{10}$ En este sentido, son varios los autores que han venido destacando la necesidad de un código que regule la buena práctica subtituladora, entre ellos, Ivarsson y Carroll que recogen un código de buenas prácticas en la subtitulación, con un total de 32 pautas generales. ${ }^{11}$ Otro autor que ha trabajado en Europa por la estandarización de la práctica traductora es Karamitroglou, ${ }^{12}$ quien propone unas directrices para la producción y distribución de subtítulos en televisión. Basándose en estos trabajos, Bartoll elabora un modelo de análisis y clasificación de los parámetros del subtitulado, distinguiendo 15 parámetros, organizados en tres categorías: Parámetros lingüísticos, pragmáticos y técnicos. ${ }^{13}$

En el caso concreto del SPS, la pluralidad de estilos en el mercado resulta aún mayor ${ }^{14}$ por ello, en España, la norma AENOR 2003 supuso un "excelente punto de

GARCÍA - Ana M ${ }^{\mathrm{a}}$ PEREIRA RODRÍGUEZ, "Subtitulado para sordos: un reto y una necesidad: pasado, presente y futuro del SPS en España”, in: J. J. Martínez Sierra (coord.), Reflexiones sobre la traducción audiovisual. Tres espectros, tres momentos, Valencia 2012, pp. 109-123.

9 El estudio se llevó a cabo por la European Association for Studies in Screen Translation (ESIST). Cfr. la página web del proyecto: http://www.esist.org/ESIST\%20Projects.htm. [consultado 16 de octubre de 2016].

10 Cfr. Jorge DÍAZ CINTAS, Teoría y Práctica de la subtitulación. Inglés-Español, Barcelona 2003, p. 138.

11 Cfr. Jan IVARSSON - Mary CARROLL, Subtitling, Simrisham 1992, pp. 157-159. Cfr. asimismo Jan IVARSSON, Subtitling for the Media: A Handbook of an Art, Stockholm 1992.

12 Cfr. su estudio de 1998, A Proposed Set of Subtitling Standards in Europe. Accessible de: http:// translationjournal.net/journal/04stndrd.htm[consultado 9 de noviembre de 2016].

13 Cfr. Eduard BARTOLL, Paramètres per a una taxonomia de la subtitulació, 2008 (accesible de: http://www.tdx.cat/bitstream/handle/10803/7572/tebt.pdf.pdf?sequence=1), así como el estudio de Jan PEDERSEN, Subtitling Norms for Television. An exploration focussing on extralinguistic cultural references, Amsterdam/Philadelphia 2011, que se centra en las normas para el subtitulado en televisión, pero haciendo especial énfasis en las referencias culturales extranlingüísticas.

14 Cfr. Verónica ARNÁIZ UZQUIZA, "La objetividad en el subtitulado. Justificación de los parámetros formales mediante Eye Tracking”, in: A. Pérez-Ugena - R. Vizcaíno-Laorga (eds.), Ulises y la comunidad sorda: hacia el desarrollo de tecnologías comunicativas para la igualdad de oportunidades, Madrid 2008, pp. 73-84, 75. 
partida para unificar criterios". ${ }^{15}$ Diversos autores han destacado las características diferenciales del SPS con respecto al subtitulado general, como son los elementos sonoros externos al diálogo, la velocidad de los subtítulos, la tipografía, etc.

"[...] it is worth considering deeper research into issues such as reading speed, readability, legibility (fonts, use of colour, positioning, ...), character identification, conveyance of sound effects or music, or at a yet more detailed approach, of the importance of conveying paralinguistic information in subtitle form. At a linguistic level, much needs to be done in the field of reduction, clarity, register or on the conveyance of metaphors and idioms, or humour, for instance." ${ }^{16}$

Los parámetros del subtitulado ordinario de Bartoll sirven de base a Arnáiz Uzquiza para la elaboración de una nueva taxonomía específica para el SPS. La autora modifica la tercera categoría de Bartoll, parámetros técnicos, que pasa a agruparse en tres: parámetros estéticos, técnicos y estético-técnicos. Añade además una sexta categoría: Parámetros extralingüísticos sonoros, que incorpora el análisis específico de los elementos sonoros que se recogen de manera exclusiva en el SPS. ${ }^{17}$

Introduciendo la variable del cambio de lengua, el subtitulado interlingüístico que encontramos en España está dirigido, en líneas generales, a espectadores oyentes. Coincidimos con Díaz Cintas cuando afirma que la división tradicional entre subtitulación interlingüística e intralingüística ha ignorado de manera sistemática los subtítulos interlingüísticos destinados a los sordos. Tal y como indica este autor:

"Se trata de una categoría híbrida que recurre a parámetros y convenciones que se usan en ambos tipos de subtitulación [...] A nivel profesional este olvido ha significado que los sordos tienen que servirse de los mismos subtítulos interlingüísticos que los oyentes normales cuando son a todas luces inapropiados para sus necesidades."18

15 Cfr. Ana M MEREIRA RODRÍGUEZ - Lourdes LORENZO GARCÍA, "Evaluamos la norma UNE 153010. Subtitulado para personas sordas y personas con discapacidad auditiva. Subtitulado a través del teletexto", Puentes 6, Granada 2005, pp. 21-26, 25.

16 Cfr. Catalina JIMÉNEZ HURTADO (ed.), Traducción y accesibilidad. Subtitulación para sordos y audiodescripción para ciegos: nuevas modalidades de Traducción Audiovisual, Frankfurt 2007, p. 30. Cfr. asimismo el estudio de Zoe DE LINDE, "Le sous-titrage intralinguistique pour les sourds et les mal entendants", in: Yves Gambier (ed.), Les transferts linguistiques dans les médias audiovisuels, Paris 1996, pp. 165-183, así como el de J. J. FUERTES - L. MARTÍNEZ, "Media Accessibility Standards in Spain", Translation Watch 3/2, Victoria 2007, pp. 61-77. Cfr. igualmente la tesis de Josélia NEVES, Audiovisual Translation: Subtitling for the Deaf and Hard-of-Hearing, Londres 2005 y su artículo de 2007: “"There is Research and Research': Subtitling for the Deaf and Hard of Hearing (SDH)".

17 Cfr. Verónica ARNÁIZ UZQUIZA, "Los parámetros que identifican el subtitulado para sordos. Análisis y clasificación”, MonTI 4, 2012, pp. 103-132, en donde la autora incluye en su detallado modelo además nuevos parámetros en algunas de las categorías, así como nuevas variables en algunos de los parámetros ya existentes (pp. 107ss.).

18 Cfr. DÍAZ CINTAS, "Teoría", p. 40. Sobre los distintos tipos de subtitulado cfr. DÍAZ CINTAS, "Teoría”, pp. 36-43 y Jorge DÍAZ CINTAS, "Nuevos retos y desarrollos en el mundo de la subtitulación", Puentes 6, 2005, pp. 13-20. 
En el siguiente apartado vamos a realizar el análisis comparado de las normas AENOR 2012 y AENOR 2003 para ver los avances que se han sucedido en este campo.

\section{Análisis de la norma UNE 153010:2012}

\subsection{Aspectos generales}

En este estudio hemos hecho una selección de aquellos aspectos que son de mayor interés para el análisis. Comenzamos comentando los aspectos generales de la norma, para centrarnos posteriormente en aquellos elementos específicos de la norma de 2012 que presentan diferencias con respecto a la anterior de 2003.

El primer rasgo general que queremos destacar es el que hace referencia al campo de aplicación de la norma $U N E$ 153010: en 2003 se ceñía al subtitulado a través del teletexto, como se hace explícito en el título: Subtitulado a través del teletexto, sin embargo, la norma de 2012 contempla todos los escenarios de comunicación audiovisual.

Otro aspecto destacable es que en 2003 se establecía que los principales usuarios de este tipo de subtitulado eran las personas sordas y las que tienen discapacidad auditiva, aunque también otros colectivos podían verse beneficiados como personas mayores, público infantil, personas que aprenden idiomas, etc. En 2012, sin embargo, se incluye también al colectivo de personas con discapacidad intelectual, lo que supone, de manera palmaria, un avance en la sensibilización de la sociedad hacia ese tipo de discapacidad no física.

Otro aspecto que queremos destacar en este apartado es que la norma de 2003 recomendaba un subtitulado adaptado para personas con dificultades de lecto-escritura. Sin lugar a duda, el colectivo de personas sordas y con discapacidad auditiva es muy heterogéneo, influido por el momento de la aparición y la detección de la pérdida auditiva, el grado de pérdida, el grado de exposición a la lengua, y la educación recibida. En este sentido, sus habilidades lectoras varían mucho y por lo tanto también sus necesidades ante el subtitulado. ${ }^{19}$ En lo relativo a este aspecto, la norma de 2012 no define criterios de calidad para elaborar subtítulos adaptados, sino para los dirigidos a un máximo de la población.

\subsection{Aspectos específicos}

Pasamos a comentar los aspectos específicos de la norma UNE 153010:2012. Para este estudio hemos agrupado los requisitos y recomendaciones en las siguientes

19 Cfr. en S. TORRES MONREAL - R. SANTANA HERNÁNDEZ, "Reading levels of Spanish deaf students", American Annals of the Deaf 150/4, Washington 2005, pp. 379-387, distintos tipos de sordos: sordos prelocutivos, personas con sordera adquirida y personas con pérdida de audición por razones de edad. Para profundizar en los diferentes perfiles y sus necesidades cfr. A. VILLALVA et al., La lectura en los sordos perlocutivos. Propuestas para un programa de entrenamiento, Madrid 2005 y NEVES, “Audiovisual Translation”, p. 26. 
categorías, siguiendo, en parte, la estructura de la norma de 2012, que se presenta más elaborada y clarificadora que la de $2003 .^{20}$

\subsubsection{Aspectos visuales de la presentación del subtítulo en pantalla}

En lo que respecta a la posición de los subtítulos, se anula la directriz de situar el texto de cada personaje debajo de la imagen, recogida en la norma de 2003. Esta estrategia había sido criticada ya que la colocación de los subtítulos debajo de cada personaje aumenta el tiempo de lectura porque el ojo tiene que desplazarse constantemente por la pantalla. ${ }^{21}$ Este cambio significa, por lo tanto, un importante avance.

En esta línea, la nueva norma apuesta porque los subtítulos de todo aquello que no sean efectos sonoros aparezcan centrados en la parte inferior de la pantalla, excepto cuando oculten información relevante. Se anula así la directriz de 2003 de diferenciar entre los subtítulos de programas en diferido, que proponía se colocaran centrados en la pantalla, mientras que los de los programas en directo irían justificados a la izquierda.

Otra novedad de la norma de 2012 es la recomendación de la presentación estática de las líneas de texto. Tal y como se indica en la misma, los estudios realizados hasta el momento desaconsejan la presentación de las líneas de texto móvil porque fija en exceso la mirada del espectador (p.9), por lo que el cambio significa asimismo un claro avance.

\subsubsection{Identificación de los personajes}

La identificación de personajes es fundamental para facilitar el seguimiento de la trama argumental. La norma de 2012 amplía la información sobre la identificación de los personajes, dedicando un capítulo aparte. Con respecto a las técnicas, se sigue priorizando la identificación mediante colores (y en concreto el uso de colores claros sobre fondos oscuros), aunque si se desaconsejase su uso, por razones técnicas o estéticas, se elegirá etiquetas o, si esto no fuera posible, guiones.

En este apartado dedicado a la identificación del personaje, la nueva norma presenta hasta siete ejemplos y seis notas aclaratorias. En líneas generales, nos parece que es un avance en la norma de 2012 el aumento de ejemplos y notas cuya finalidad es recoger casos que presentan problemas al subtitulador y proponer soluciones claras. Los investigadores habían hecho hincapié precisamente en esta necesidad de que la norma recogiera soluciones a los posibles problemas con los que tenía que enfrentarse el subtitulador. ${ }^{22}$

En este sentido, la norma de 2012 aporta directrices de gran utilidad para los subtituladores sobre la posición y la edición de las etiquetas para identificar a los personajes: deben anteceder al subtítulo y estar escritas en mayúscula y entre paréntesis:

20 Los epígrafes de la AENOR 2012 son: Aspectos visuales de la presentación del subtítulo en pantalla, Aspectos temporales, Identificación de los personajes, Efectos sonoros, Información contextual y voz en off, Música y canciones y, por último, Criterios editoriales.

21 Cfr. PEREIRA RODRÍGUEZ - LORENZO GARCÍA, "Evaluamos la norma”, p. 22.

22 Este era uno de los aspectos que destacaban PEREIRA RODRÍGUEZ - LORENZO GARCÍA al evaluar la norma de 2003 (p. 22). 
EJEMPLO 1 (RAFAEL) ¡Hola, amigos!

EJEMPLO 2 (ANCIANA) Hoy hace mucho frío.

También se aborda el uso de abreviaturas en las etiquetas: la primera vez que aparece el personaje se escribe el nombre completo seguido de un guión y de la abreviatura que se haya escogido (todo ello en mayúscula y entre paréntesis), y posteriormente se emplea la abreviatura:

EJEMPLO 1 La primera intervención de Marta se subtitularía: (MARTA-M) Texto

NOTA 1 A partir de ahí no será necesario utilizar el nombre completo sino la abreviatura

EJEMPLO 2 El ejemplo anterior se subtitularía (MT) Texto

NOTA 2 Cuando dos nombres empiecen por la misma letra tendrían que tomar aquella que los diferencie o caracterice. (UNE 153010:2012, p. 12).

En lo que respecta al contraste entre caracteres y su contorno o caja, una importante novedad de 2012 es que introduce un anexo de tres páginas que recoge las medidas relacionadas con el uso del color, en el que se detalla cómo calcular la diferencia entre los colores, así como la medida de relación de contraste. En este sentido, la norma de 2012 está más elaborada, recoge más tecnicismos. ${ }^{23}$

\subsubsection{Aspectos temporales de la presentación del subtítulo en pantalla}

En lo relativo a la velocidad de exposición del texto del subtítulo, la norma de 2003 recomendaba en el teletexto 19 caracteres por segundo (cps), partiendo de una velocidad de 150 palabras por minuto y, para subtítulos adaptados, $12 \mathrm{cps}$, teniendo como base un ritmo de lectura de 95 palabras por minuto. La norma de 2012, con el intento de satisfacer a los dos tipos de receptores, propone 15 cps como máximo. Esta directriz supone un avance: 4,66 segundos para la lectura de los 70 caracteres (en dos líneas), es decir, se ha llegado a la regla de los cuatro segundos, pero no a la de seis segundos que recomiendan los investigadores. ${ }^{24}$

En lo que respecta al sincronismo, la norma de 2012 recoge dos anexos de seis páginas en total, dedicados a los cálculos de precisión de los subtítulos en directo y el retardo de los subtítulos en directo ${ }^{25}$ mientras que en 2003 se dedicaba al tema menos de media página. ${ }^{26}$ Precisamente, la principal fuente de reclamaciones de los usuarios del subtitulado en España es la falta de sincronización en el subtitulado en directo, lo que supone uno de los más importantes retos tecnológicos pendientes en el ámbito de la televisión. ${ }^{27}$

23 Cfr. norma UNE 153010:2012, pp. 20-22.

24 Cfr. la propuesta en favor de los seis segundos en PEREIRA RODRÍGUEZ - LORENZO GARCÍA, "Evaluamos la norma", p. 23.

25 Cfr. norma UNE 153010:2012, pp. 23-28.

26 Cfr. norma Cfr. norma UNE 153010:2003, p. 10.

27 Cfr. "Líneas de investigación en el Centro Español del Subtitulado y la Audiodescripción (CESyA)", FIAPAS 144, 2013, pp. 16-17. 


\subsubsection{Efectos sonoros}

Otro aspecto específico del SPS son los elementos sonoros externos al diálogo, por ello es conveniente destacar en este punto que la norma de 2012 define con mayor precisión lo que se considera un efecto sonoro, ampliando la definición:

Tab. 1 Efectos sonoros.

\begin{tabular}{|l|l|}
\hline \multicolumn{1}{|c|}{ AENOR 2003: 4 } & \multicolumn{1}{c|}{ AENOR 2012: 5 } \\
\hline $\begin{array}{l}\text { "Sonidos que se } \\
\text { producen en una } \\
\text { película o programa" }\end{array}$ & $\begin{array}{l}\text { "Sonido no vocal (exceptuando el habla y que no se pueda } \\
\text { atribuir a un personaje concreto) que aporta información } \\
\text { relevante para el seguimiento de la obra audiovisual." }\end{array}$ \\
\hline
\end{tabular}

Además AENOR 2012 introduce aclaraciones y ejemplos en los casos de subtitulos grabados, subtítulos directos y semi-directos, algo que no aparecía en la norma de 2003, favoreciendo la labor del subtitulador. ${ }^{28}$

\subsubsection{Música y canciones}

Otros elementos sonoros externos al diálogo que merecen especial atención en el SPS son la música y las canciones. ${ }^{29}$ Se observa un claro avance entre ambas normas: La norma de 2012 dedica un capítulo aparte a este tema con el epígrafe "Música y canciones", dando una mayor relevancia al tema, y afirma que se debería subtitular la letra de las canciones si es importante para ayudar al espectador a comprender la trama. En la norma de 2012 se anula la única directriz que proporcionaba la norma de 2003 en relación a las canciones: debían subtitularse las canciones y que ello se debía realizarse con caracteres azules sobre fondo amarillo. ${ }^{30}$

También supone un avance la norma de 2012 al especificar el marcado de las canciones con la siguiente directriz: la manera de marcarlo es usando el símbolo de una nota musical (o almohadilla en el caso de que la tecnología no lo permita). ${ }^{31}$

\subsubsection{Criterios editoriales}

La norma de 2012 añade un último epígrafe en el que bajo "Criterios editoriales" recoge directrices que, en parte, ya aparecían en la norma de 2003, pero ahora están mejor estructuradas. De manera ilustrativa aportamos algunos ejemplos de ambas normas en contraste. Como se observa, aumenta el mayor número de ejemplos y notas, así como las estrategias para economizar en el vocabulario:

\footnotetext{
28 Cfr. norma UNE 153010:2012, pp. 13-14.

29 En el caso de musicales, las canciones formarían parte del diálogo.

30 Cfr. norma UNE 153010:2003, p. 14.

31 Cfr. norma UNE 153010:2012, pp. 15-16.
} 
Uso de abreviaturas, siglas, símbolos y acrónimos.

Tab. 2 Criterios editoriales.

\begin{tabular}{|l|l|}
\hline \multicolumn{1}{|c|}{ AENOR 2003: 12 } & \multicolumn{1}{c|}{ AENOR 2012: 9 } \\
\hline Otan, Onu & $\begin{array}{l}\text { - CC.OO., EE.UU-Bonu, Unicef, Renfe, ovnbi, sida... } \\
\text { - Estamos a 25 km de nuestro destino. } \\
\\
\end{array}$ \\
\hline
\end{tabular}

Tab. 3 Eliminación de información superflua, como muletillas y repeticiones.

\begin{tabular}{|l|l|}
\hline \multicolumn{1}{|c|}{ AENOR 2003 } & \multicolumn{1}{c|}{ AENOR 2012: 29 } \\
\hline [sin ejemplo] & - Bueno, ¿Cómo te ha ido?/¿Cómo te ha ido? \\
& $-¡$ ¿Vamos, Pepe, venga!/¡Vamos, Pe pe! \\
\hline
\end{tabular}

Tab. 4 Uso de pronombres.

\begin{tabular}{|l|l|}
\hline \multicolumn{1}{|c|}{ AENOR 2003 } & \multicolumn{1}{c|}{ AENOR 2012: 29 } \\
\hline [sin ejemplo] & - EJEMPLO \\
& $\begin{array}{l}\text { Este esel coche de José Antonio/Este es su coche./Es su coche. } \\
\text { - La casa de Pablo es más grande que la casa de María/ Su } \\
\text { casa es más grande que la de María/Su casa es más grande } \\
\text { que la de ella. }\end{array}$ \\
\hline
\end{tabular}

Tab. 5 Reducción en nombres de entidades, organismos y cargos.

\begin{tabular}{|l|l|}
\hline \multicolumn{1}{|c|}{ AENOR 2003: 12 } & \multicolumn{1}{c|}{ AENOR 2012: 29 } \\
\hline $\begin{array}{l}\text { "La Cámara de los } \\
\text { diputados" por "El } \\
\text { Congreso" }\end{array}$ & EJEMPLO \\
& $\begin{array}{l}\text { Sus Majestades los Reyes de España / SS.MM. Los Reyes. } \\
\text { Isabel la Catolica y Fernando el Católico / Los Reyes } \\
\text { Católicos. } \\
\text { Cámara de los senadores / El Senado. } \\
\\
\text { Su Santidad Benedicto XVI / El Papa. }\end{array}$ \\
\hline
\end{tabular}

Tab. 6 Uso del plural genérico para evitar la repetión de términos en ambos géneros.

\begin{tabular}{|c|l|}
\hline AENOR 2003 & \multicolumn{1}{c|}{ AENOR 2012: 29 } \\
\hline [no se recoge estrategia] & $\begin{array}{l}\text { Los alumnas / Los alumnos } \\
\text { Quienes estén capacitados y capacitadas / Quienes están } \\
\text { capacitados (2012: 29) }\end{array}$ \\
\hline
\end{tabular}

Tab. 7 Simplificación de formas verbales.

\begin{tabular}{|c|l|}
\hline AENOR 2003 & \multicolumn{1}{c|}{ AENOR 2012: 29 } \\
\hline [no se recoge estrategia] & $\begin{array}{l}\text { ¿Cómo te has enterado?/¿Cómo te enteraste? } \\
\text { Me habían comentado que no ibas a venir / Me comentaron } \\
\text { que no vendrías. (2012: 29) }\end{array}$ \\
\hline
\end{tabular}




\section{Consideraciones finales}

Tras este análisis queremos destacar, en primer lugar, que los avances que se observan en el camino hacia la eliminación de barreras son un claro reflejo de la creciente sensibilización de la sociedad hacia la discapacidad. A través del análisis comparado se constata que la norma de 2012, fruto de una labor de consenso entre los distintos actores implicados, introduce más tecnicismos y añade un mayor número de ejemplos y notas, proponiendo soluciones a los posibles problemas que puedan surgir, lo que facilita la labor del subtitulador.

Sin lugar a duda, para poder desarrollar esa tarea de subtitulación, para poder hacer frente a ese desafío, se necesitará a unos profesionales con una formación de calidad que debe venir proporcionada por las universidades. ${ }^{32}$

Pero además de la formación, la investigación en el ámbito académico será una pieza fundamental para proporcionar a la sociedad estudios empíricos que contribuyan al desarrollo de las normativas..$^{33}$ En el caso que nos ocupa, serán necesarios estudios que permitan evaluar la adecuación de los parámetros que recoge la norma de 2012. Precisamente la dificultad de la estandarización de los subtítulos para sordos radica no solo en elaborar los parámetros que sean más apropiados, sino también en la heterogeneidad de la audiencia meta. En este sentido, serán necesarios estudios que contribuyan a facilitar de manera más precisa el perfil de la audiencia y sus necesidades a la hora de consumir productos audiovisuales.

Estos logros que se han conseguido a nivel institucional en el campo del SPS, con la aprobación de leyes y normativas deben ir acompañados de una investigación desde las áreas del saber implicadas, entre ellas, la Lingüística y la Traductología. Los numerosos trabajos de interés que se han publicado en los últimos años ${ }^{34}$

32 La primera línea de actuación del I Plan Nacional de Accesibilidad (2004-2012) ha sido precisamente la concienciación y la formación, estableciendo una serie de objetivos tendentes a la consecución de la Accesibilidad Universal, a través de la generalización del Diseño para Todos. Bajo el lema: Universidad sin barreras, la UNED publicó en 2012 una Guía de accesibilidad de documentos electrónicos, cfr. Valentín SAMA ROJO - Esther SEVILLANO ASENSIO, Guía de accesibilidad de documentos electrónicos, UNED, 2012. Accesible de: http://portal.uned.es/portal/page?_pageid $=93,26066088 \&$ \&dad=portal\&_schema=PORTAL [consultado el 20 de octubre de 2016].

33 Cfr. Investigación: Observatorio 'Universidad y Discapacidad'. Fundación Universia y CERMI (Comité Español de Representantes de Personas con Discapacidad), FIAPAS 146, 2013, pp. 22-23.

34 Cfr. en Anna MATAMALA - Pilar ORERO (eds.), Listening to Subtitles: Subtitles for the Deaf and Hard of Hearing, Frankfurt 2010, los trabajos de, entre otros, Francisco UTRAY et al., "Maximum font size for subtitles in Standard Definition Digital Television: Tests for a font magnifying application", Eduard BARTOLL - Anna MARTÍNEZ TEJERINA, "The positioning of subtitles for the deaf and hard of hearing", Ana $\mathrm{M}^{\mathrm{a}}$ PEREIRA RODRÍGUEZ, "Criteria for elaborating subtitles for deaf and hard of hearing adults in Spain: Description of a case study", Ana Ma PEREIRA RODRÍGUEZ, "Including Spanish Sign Language in subtitles for deaf and hard of hearing", Lourdes LORENZO GARCÍA, "Subtitling for deaf and hard of hearing children in Spain: A case study", Lourdes LORENZO GARCÍA "Criteria for elaborating subtitles for deaf and hard of hearing children in Spain: A guide of good practice", Clara CIVERA - Pilar ORERO,"Introducing icons in subtitles for deaf and hard of hearing: Optimising reception?", Verónica ARNÁIZ UZQUIZA, "SUBSORDIG: The need for a deep analysis of data" y Ana Ma PEREIRA RODRÍGUEZ - Verónica ARNÁIZ UZQUIZA, "A comprehensive bibliography on subtitling for the deaf and hard of 
auguran un futuro de esperanza en este camino hacia la eliminación de barreras para discapacitados, en definitiva, en este camino hacia una sociedad más justa, hacia una sociedad más inclusiva.

(Escrito en español por la autora)

\section{BIBLIOGRAFÍA}

AENOR, Asociación Española de Normalización y Certificación (2003): Norma UNE 153010:2003 Subtitulado para personas sordas y personas con discapacidad auditiva. Subtitulado a través del teletexto, Madrid: Asociación Española de Normalización y Certificación.

AENOR, Asociación Española de Normalización y Certificación (2012): Norma UNE 153010:2012. Subtitulado para personas sordas y personas con discapacidad auditiva, Madrid: Asociación Española de Normalización y Certificación.

ARNÁIZ UZQUIZA, Verónica,"La objetividad en el subtitulado. Justificación de los parámetros formales mediante Eye Tracking", in: A. Pérez-Ugena - R. Vizcaíno-Laorga (eds.), Ulises y la comunidad sorda: hacia el desarrollo de tecnologías comunicativas para la igualdad de oportunidades, Madrid: Asociación Observatorio de las Realidades Sociales y de la Comunicación, 2008, pp. 73-84.

ARNÁIZ UZQUIZA, Verónica, "SUBSORDIG: The need for a deep analysis of data", in: Anna Matamala - Pilar Orero (eds.), Listening to Subtitles: Subtitles for the Deaf and Hard of Hearing, Frankfurt: Peter Lang, 2010, pp. 163-174.

ARNÁIZ UZQUIZA, Verónica, "Los parámetros que identifican el subtitulado para sordos. Análisis y clasificación”, in: MonTI 4, Alicante: Universidad de Alicante, 2012, pp. 103-132.

BADIA, Toni - MATAMALA, Anna, "La docencia en accesiblidad en los medios", in: Trans. Revista de traductología II, Málaga: Universidad de Málaga 2007, pp. 61-71.

BARTOLL, Eduard, Paramètres per a una taxonomia de la subtitulació, 2008. Accesible de: http:// www.tdx.cat/bitstream/handle/10803/7572/tebt.pdf.pdf?sequence=1 [consultado 9 de noviembre de 2016]

BARTOLL, Eduard - MARTÍNEZ TEJERINA, Anjana, "The positioning of subtitles for the deaf and hard of hearing", in: Anna Matamala - Pilar Orero (eds.), Listening to Subtitles: Subtitles for the Deaf and Hard of Hearing, Frankfurt: Peter Lang, 2010, pp. 69-86.

CIVERA, Clara - ORERO, Pilar, "Introducing icons in subtitles for deaf and hard of hearing: Optimising reception?", in: Anna Matamala - Pilar Orero (eds.), Listening to Subtitles: Subtitles for the Deaf and Hard of Hearing, Frankfurt: Peter Lang, 2010, pp. 149-162.

CUÉLLAR LÁZARO, Carmen, "Kulturspezifische Elemente und ihre Problematik bei der Filmsynchronisierung”, in: Journal of Arts \& Humanities 2/6, Annapolis: Maryland Institute of Research, 2013, pp. 134-146.

hearing from a multidisciplinary approach". Este último reúne un interesante repertorio bibliográfico sobre el subtitulado para personas sordas o con discapacidad auditiva. Cfr. también el estudio de Agnieszka SZARKOWSKA et al., "Subtitling for the deaf and hard of hearing in multilingual films", International Journal of Multilingualism 10/3, Abingdon 2013, pp. 292-312, así como el trabajo de Pablo ROMERO FRESCO, Subtitling Through Speech Recognition: Respeaking, Manchester 2011. Finalmente cabe destacar la obra que presenta el Proyecto Ulises (Utilización Lógica e Integrada del sistema Europeo de Signos/ Señas), publicada por A. PÉREZ-UGENA R. VIZCAÍNO-LAORGA, "Ulises”, con el estudio de Anjana MARTÍNEZ TEJERINA, "El tamaño importa y la posición también. Estudio piloto sobre el formato de los subtítulos para sordos" y el trabajo de Pablo ROMERO FRESCO, "La subtitulación rehablada: Palabras que no se lleva el viento". Cfr. asimismo el proyecto iberoamericano Red Melisa (Red para la mejora de la calidad de servicios interactivos y accesibilidad en la TDT para reducir la brecha digital) http://www. redmelisa.org/ 
CUÉLLAR LÁZARO, Carmen, "El subtitulado para sordos en España y Alemania: estudio comparado de los marcos normativos y la formación universitaria", in: Revista Española de Discapacidad 4/2, Madrid: Centro Español de Documentación sobre Discapacidad (CEDD): 12016.

DE LINDE, Zoe, "Le sous-titrage intralinguistique pour les sourds et les mal entendants", in: Yves Gambier (ed.), Les transferts linguistiques dans les médias audiovisuels, Paris: Presses Universitaires du Septentrion, 1996, pp. 165-183.

DÍAZ CINTAS, Jorge, Teoría y Práctica de la subtitulación. Inglés-Español, Barcelona: Ariel, 2003.

DÍAZ CINTAS, Jorge, "Nuevos retos y desarrollos en el mundo de la subtitulación”, in: Puentes 6, Granada: Universidad de Granada 2005, pp. 13-20

FUERTES, J. L./MARTÍNEZ, L., "Media Accessibility Standards in Spain”, in: Translation Watch 3/2, Victoria: Translation Standards Institute, 2007, pp. 61-77.

IVARSSON, Jan, Subtitling for the Media: A Handbook of an Art, Stockholm: TransEdit, 1992.

IVARSSON, Jan - Mary CARROLL, Subtitling, Simrisham: TransEdit HB, 1998.

JIMÉNEZ HURTADO, Catalina (ed.), Traducción y accesibilidad. Subtitulación para sordos y audiodescripción para ciegos: nuevas modalidades de Traducción Audiovisual, Frankfurt: Peter Lang, 2007.

KARAMITROGLOU, Fotios, "A proposed set of subtitling standards in Europe”, in: Translation Journal 2/2, Palatine: Translationz Corp.1998. Accesible de: http://translationjournal.net/journal/04stndrd .htm ). [consultado el 9 de noviembre de 2016]

LORENZO GARCÍA, Lourdes, "Subtitling for deaf and hard of hearing children in Spain: A case study", in: Anna Matamala - Pilar Orero (eds.), Listening to Subtitles: Subtitles for the Deaf and Hard of Hearing, Frankfurt: Peter Lang, 2010a, pp. 115-138.

LORENZO GARCÍA, Lourdes, "Criteria for elaborating subtitles for deaf and hard of hearing children in Spain: A guide of good practice", in: Anna Matamala - Pilar Orero (eds.), Listening to Subtitles: Subtitles for the Deaf and Hard of Hearing, Frankfurt: Peter Lang, 2010b, pp. 139-148.

LORENZO GARCÍA, Lourdes - PEREIRA RODRÍGUEZ, Ana M"a "Subtitulado para sordos: un reto y una necesidad: pasado, presente y futuro del SPS en España", in: J.J. Martínez Sierra (coord.), Reflexiones sobre la traducción audiovisual. Tres espectros, tres momentos, Valencia: Universitat de Valencia, 2012, pp. 109-123.

MARTÍNEZ TEJERINA, Anjana 2008: El tamaño importa y la posición también. Estudio piloto sobre el formato de los subtítulos para sordos, in: A. Pérez-Ugena - R. Vizcaíno-Laorga (eds.), Ulises y la comunidad sorda: hacia el desarrollo de tecnologías comunicativas para la igualdad de oportunidades, Madrid: Asociación Observatorio de las Realidades Sociales y de la Comunicación, pp. 85-92.

MATAMALA, Anna - ORERO, Pilar (eds.), Listening to Subtitles: Subtitles for the Deaf and Hard of Hearing, Frankfurt: Peter Lang, 2010.

NEVES, Josélia, Audiovisual Translation: Subtitling for the Deaf and Hard-of-Hearing, Londres: University of Surrey Roehampton, 2005. Tesis inédita.

NEVES, Josélia, “'There is research and research': Subtitling for the Deaf and Hard of Hearing (SDH)”, in: Catalina Jiménez Hurtado (ed.), Traducción y accesibilidad. Subtitulación para sordos y audiodescripción para ciegos: nuevas modalidades de Traducción Audiovisual, Frankfurt: Peter Lang, 2007.

NEVES, Josélia - LORENZO GARCÍA, Lourdes, "La subtitulación para s/Sordos, panorama global y prenormativo en el marco ibérico", in: Trans. Revista de traductología 11, Málaga: Universidad de Málaga, 2007, pp. 95-114.

ORERO, Pilar, "Visión histórica de la accesibilidad en los medios en España", in: Trans. Revista de traductología II, Málaga: Universidad de Málaga, 2007, pp. 31-43.

PEDERSEN, Jan, Subtitling Norms for Television. An Exploration Focussing on Extralinguistic Cultural References, Amsterdam/Philadelphia: John Benjamins Publishing Company, 2011.

PEREIRA RODRÍGUEZ, Ana Ma " "El subtitulado para sordos: estado de la cuestión en España", Quaderns 11, Barcelona: Universidad Autónoma de Barcelona 2005, pp. 161-172. 
PEREIRA RODRÍGUEZ, Ana $\mathrm{M}^{\mathrm{a}}$, "Criteria for elaborating subtitles for deaf and hard of hearing adults in Spain: Description of a case study", in: Anna Matamala - Pilar Orero (eds.), Listening to Subtitles: Subtitles for the Deaf and Hard of Hearing, Frankfurt: Peter Lang, 2010a, pp. 87-103.

PEREIRA RODRÍGUEZ, Ana M"a "Including Spanish Sign Language in subtitles for deaf and hard of hearing", in: Anna Matamala - Pilar Orero (eds.), Listening to Subtitles: Subtitles for the Deaf and Hard of Hearing, Frankfurt: Peter Lang, 2010b, pp. 103-114.

PEREIRA RODRÍGUEZ, Ana $\mathrm{M}^{\mathrm{a}}$ - LORENZO GARCÍA, Lourdes, "Evaluamos la norma UNE 153010. Subtitulado para personas sordas y personas con discapacidad auditiva. Subtitulado a través del teletexto", in: Puentes 6, Granada: Universidad de Granada 2005, pp. 21-26.

PEREIRA RODRÍGUEZ, Ana M ${ }^{\mathrm{a}}$ - ARNÁIZ UZQUIZA, Verónica, "A comprehensive bibliography on subtitling for the deaf and hard of hearing from a multidisciplinary approach, in: Anna Matamala - Pilar Orero (eds.), Listening to Subtitles: Subtitles for the Deaf and Hard of Hearing, Frankfurt: Peter Lang, 2010a, pp. 219-227.

PÉREZ-UGENA, A. - VIZCAÍNO-LAORGA, R. (eds.), Ulises y la comunidad sorda: hacia el desarrollo de tecnologías comunicativas para la igualdad de oportunidades, Madrid: Asociación Observatorio de las Realidades Sociales y de la Comunicación, 2008.

ROMERO FRESCO, Pablo, "La subtitulación rehablada: Palabras que no se lleva el viento", in: A. Pérez-Ugena - R. Vizcaíno-Laorga (eds.), Ulises y la comunidad sorda: hacia el desarrollo de tecnologías comunicativas para la igualdad de oportunidades, Madrid: Asociación Observatorio de las Realidades Sociales y de la Comunicación, 2008, pp. 49-72.

ROMERO FRESCO, Pablo, Subtitling Through Speech Recognition: Respeaking, Manchester: St. Jerome Publishing, 2011.

SAMA ROJO, Valentín - SEVILLANO ASENSIO, Esther. Guía de accesibilidad de documentos electrónicos, UNED, 2012. Accesible de: http://portal.uned.es/portal/page?_pageid=93,26066088\& _dad=portal\&_schema=PORTAL [consultado el 20 de octubre de 2016].

SZARKOWSKA, Agnieszka-ŻBIKOWSKA, Jagoda-KREJTZ, Izabela, "Subtitling for the deaf and hard of hearing in multilingual films", in: International Journal of Multilingualism 10/3, 2013, pp. 292-312. Accesible de: http://dx.doi.org/10.1080/14790718.2013.766195 [consultado el 20 de octubre de 2016].

TORRES MONREAL, S. - SANTANA HERNANDEZ, R., "Reading levels of Spanish deaf students", in: American Annals of the Deaf 150 (4), Washington: Gallaudet University Press 2005, pp. 379-387.

UTRAY, Francisco - PEREIRA RODRÍGUEZ, Ana M ${ }^{\mathrm{a}}$ - ORERO, Pilar, "The Present and Future of Audio Description and Subtitling for the Deaf and Hard of Hearing in Spain", in: Meta 54/2, Montréal: Les Presses de l'Université de Montréal, 2009, pp. 248-263.

UTRAY, Francisco - RUIZ, Belén - MOREIRO, José Antonio, "Maximum font size for subtitles in Standard Definition Digital Television: Tests for a font magnifying application", in: Anna Matamala - Pilar Orero (eds.), Listening to Subtitles: Subtitles for the Deaf and Hard of Hearing, Frankfurt: Peter Lang, 2010, pp. 59-68.

VILLALBA, A. et al., La lectura en los sordos perlocutivos. Propuestas para un programa de entrenamiento, Madrid: Entha Ediciones, 2005.

\section{Información breve sobre la autora}

La Dra. Carmen Cuéllar Lázaro defendió su tesis doctoral en la Friedrich-Schiller Universität Jena, Jena (Alemania). En esta institución compaginó docencia e investigación con un plaza de Ayudante de Investigación. Posteriormente fue Profesora Titular en la Facultad de Traducción e Interpretación de la Universidad de Valladolid, donde desempeñó a su vez el cargo de Secretaria Académica. En la actualidad es Directora del Departamento de Filología Francesa y Alemana en la Facultad de Filosofía y Letras.

Ha realizado distintas estancias de estudio e investigación en universidades de Alemania, Austria y Reino Unido. Asimismo ha participado en varios proyectos de 
investigación e innovación financiados y ha publicado numerosos capítulos de libros y artículos en revistas de carácter internacional, relacionados con sus líneas de investigación.

Forma parte del Grupo de Investigación de Excelencia de la Universidad de Valladolid ITNT (Intersemiótica, Traducción y Nuevas Tecnologías), y es miembro de varios comités científicos de revistas internacionales, así como de la prestigiosa institución alemana Deutsche Akademische Austauschdienst (DAAD).

Correo electrónico: carmen.cuellar@lesp.uva.es 